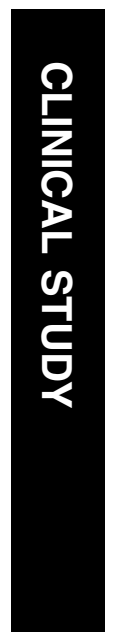

\title{
Visual complications of proton beam therapy for clival chordoma
}

\begin{abstract}
Purpose Combining maximal surgical resection with high-dose proton radiation therapy is reported to be currently the best management of patients with clival chordoma. Method Since 1991, four consecutive patients from our institution with this tumour have been referred for postoperative proton beam radiotherapy.

Result We have experienced an unusually high complication rate $\mathbf{5 0 \%}$ ) of delayed radiation optic neuropathy. This has resulted in profound, bilateral visual loss at 1 and 2 years postproton beam treatment.

Conclusion It has served as a reminder that proton beam therapy is not an innocuous treatment option and this devastating complication should be taken into account when discussing the management of clivus chordoma. We postulate whether the optic apparatus is particularly sensitive to proton vs photon damage.

Eye (2003) 17, 318-323. doi:10.1038/

sj.eye. 6700339
\end{abstract}

'Department of Ophthalmology Walton Hospital Liverpool, UK

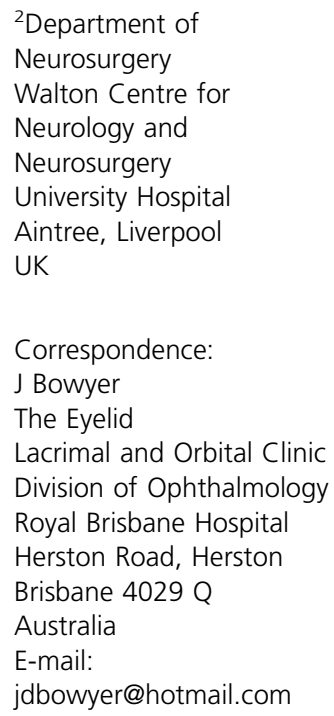

Received: 30 April 2001 Accepted in revised form: 10 June 2002

Accepted for poster presentation Annual Congress 2001, Royal College of Ophthalmologists
Keywords: radiation optic neuropathy; proton beam therapy; clival chordoma

\section{Introduction}

Chordomas are embryonal tumours derived from residues of primitive notochordal cells in the axial skeleton. Deeply located at the centre of the skull base, the clivus chordoma is a rare but extremely difficult management problem. Growing slowly but locally invasive, complete surgical resection is nearly impossible. Most patients succumb to recurrent disease. Combining maximal surgical resection with high-dose proton radiation therapy has been reported to be currently the best management of these patients. ${ }^{1}$ We are unaware that the neurovisual outcome of patients treated for clival chordoma with proton-photon therapy
J Bowyer', S Natha', I Marsh' and P Foy ${ }^{2}$

has been reported in detail in the ophthalmic literature.

\section{Materials and methods}

Between 1991 and 1997, following subtotal resection of a clival chordoma, four consecutive patients were referred for postoperative highdose combined proton/photon beam therapy.

\section{Results}

Case 1

A 55-year-old female presented to the neurologists with a history of nasal obstruction, occipital and posterior upper neck pain, speech problems, and diplopia. On examination left VIth and right IXth, Xth and XIth cranial nerve palsies were found. There was a history of hypertension controlled on treatment. Magnetic resonance imaging (MRI) revealed a large clival tumour extending into the retropharyngeal space and displacing the basilar artery (Figure 1).

Radical resection of the chordoma was performed through maxillotomies with division of the soft palate. The tumour was extending from the foramen magnum to the sphenoid and was excised macroscopically. The defect was repaired with a free-radial forearm flap. Histological examination confirmed a chordoma.

Following ophthalmic review, botulinum toxin was injected into the left medial rectus. Visual acuity at this time was recorded as 6/9 bilaterally.

Residual tumour was noted on follow-up MRI scanning at 9 months postsurgery and the patient was referred for proton beam therapy (Figure 2).

Pretreatment planning scans confirmed recurrence of disease behind the upper clivus and right cerebellopontine angle but not involving the optic pathway. 


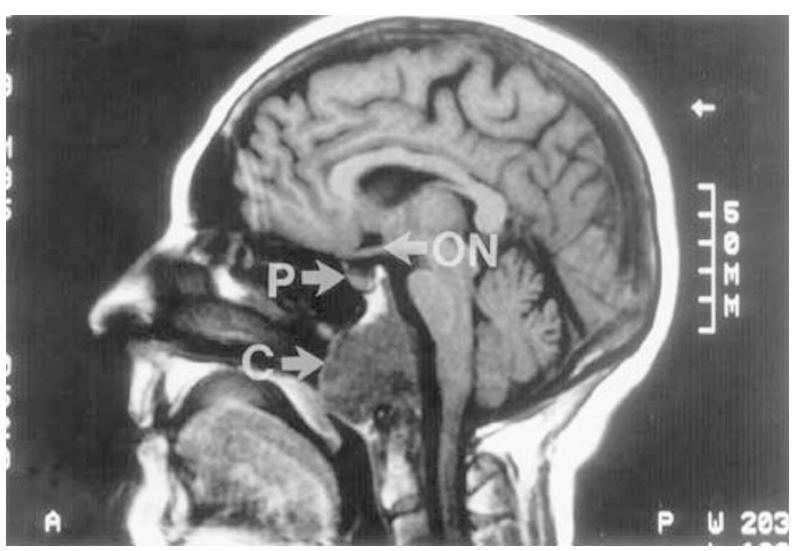

Figure 1 Preoperative sagital T1-weighted MRI scan without gadolinium enhancement (arrows to chordoma (C), optic nerve $(\mathrm{ON})$, and pituitary $(\mathrm{P}))$.

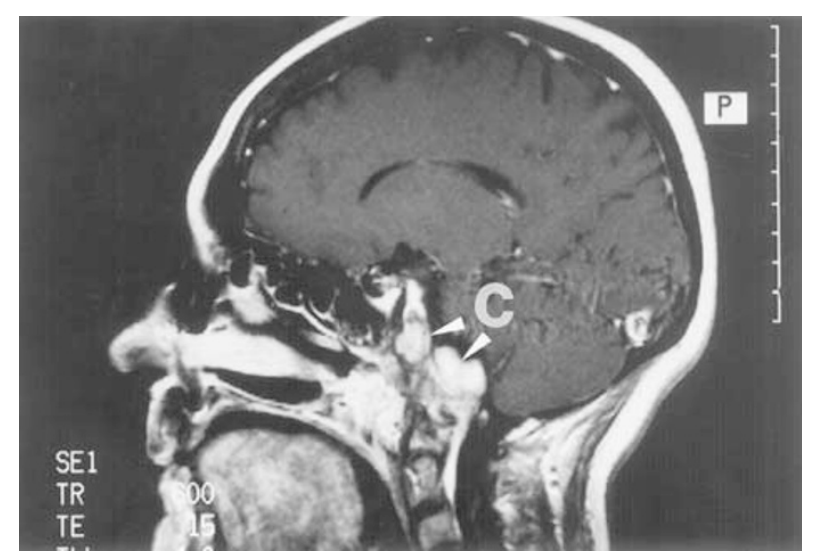

Figure 2 T1-weighted axial MRI scan with gadolinium enhancement showing postoperative evidence of residual tumour $(\mathrm{C})$ in the clivus and foramen magnum prior to proton beam radiotherapy.

Proton/photon beam therapy was undertaken delivering 75.5 cobalt gray equivalent (CGE) to the tumour in 40 fractions (photon dose 19.8 Gy in 11 fractions and proton dose 55.7CGE in 29 fractions) (Figure 3).

One year posttreatment, the patient presented to us with blurred vision. Visual acuities of $6 / 9$ right and 6/60 left were recorded with a left relative afferent defect. Repeat scanning showed no hydrocephalus or tumour extension. There was a small area of enhancement in front of the brainstem. Within 2 weeks, the vision dropped to $3 / 24$ on the right side and HM on the left. Colour vision was not recordable and the pupils were sluggish. Dexamethasone $4 \mathrm{mg}$ QDS was commenced for 10 days without effect. The optic discs became progressively pale (Figure 4).

Visual acuities of CF and HM with a right RAPD were recorded at the last visit.
A right VIIth nerve palsy developed 18 months posttreatment. The patient has since required two right lateral tarsorrhaphies and a botulinum toxin ptosis for inferior corneal exposure and an episode of microbial keratitis.

A severe radionecrosis of the temporal lobes was noted 2 years after treatment. There was a slow response to a 2-month course of systemic steroids.

Pituitary function has remained normal.

\section{Case 2}

A 54-year-old female described a 3-year history of worsening imbalance and gait unsteadiness. She developed difficulty with finding words, pronunciation, short-term memory, and numbers. There were no ophthalmic symptoms or signs. MRI revealed a tumour of the upper clivus, eroding and destroying bone and compressing the brainstem. A radiological diagnosis of chordoma was made.

A right subtemporal transtentorial approach to the tumour was carried out. The lesion was found to be closely adherent to the brainstem and invaded the right cavernous sinus precluding total resection. An intradural chondroid chordoma was confirmed on histology.

Postoperatively there was weakness of the right fourth nerve. Imaging revealed residual tumour of the clivus with extension into the right and left cavernous sinus region (Figure 5). The optic nerves and chiasm though were free of tumour (Figure 6).

It was felt that optimum curative treatment would be achieved with a combination of high-dose protons and photons. Proton beam therapy was undertaken to a total dose of 83.5CGE (photon dose 14.4 Gy in eight fractions and proton dose 69.1 CGE in 36 fractions).

Three months following treatment, the patient developed radiation-induced inhibition of the hypothalamic/pituitary axis. She commenced hydrocortisone and thyroxine replacement.

At an ophthalmic review 15 months following this, visual acuities of $6 / 12$ bilaterally were documented. Two months later she experienced sudden loss of vision to $6 / 24$ (N12) on the right and 6/36 on the left. A left relative afferent defect was noted with loss of colour vision and a pale left optic disc. Delayed radionecrosis of the optic nerve was diagnosed. No sign of tumour recurrence has been found on repeat scanning (Figure 7).

At last review, visual acuity was 6/36 (N48) right eye with a superior field defect and 6/60 left with global loss on Henson field testing.

\section{Case 3}

A 70-year-old female presented 3 years ago with two episodes of bacterial meningitis and cerebrospinal fluid 

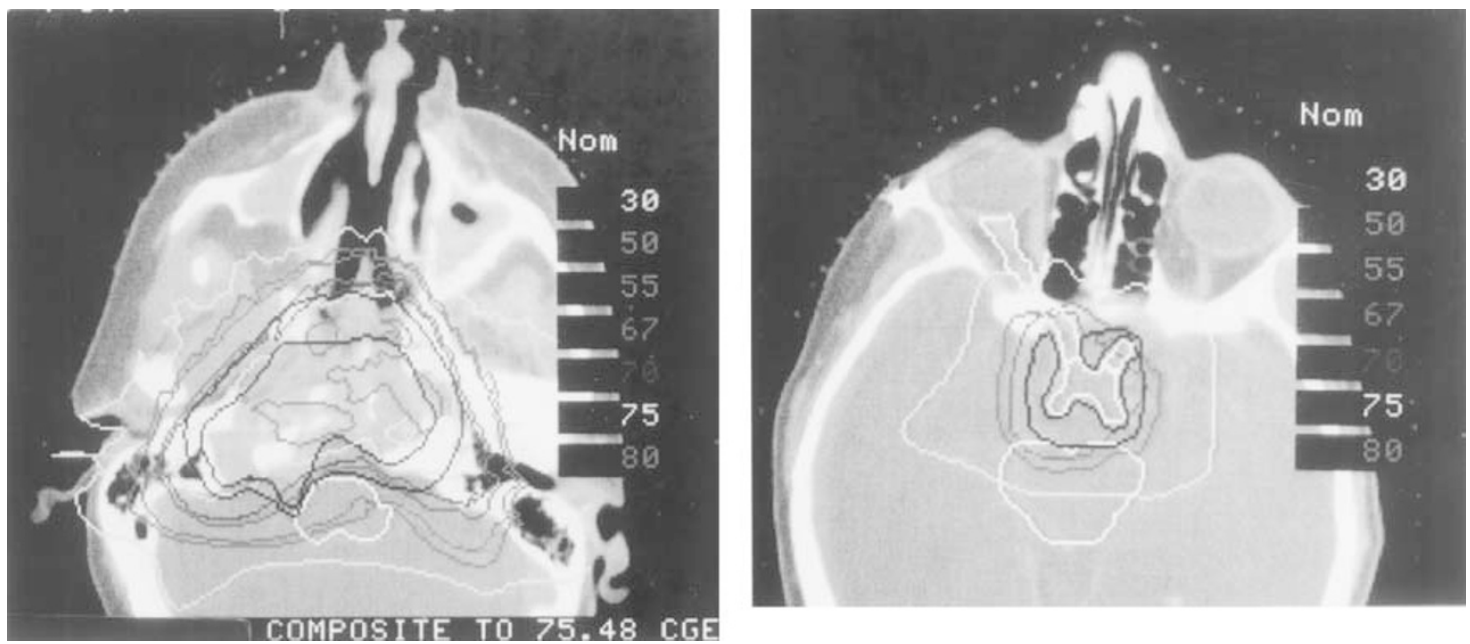

Figure 3 Computer tomography scans showing the dose distribution to the chordoma at the skull base (left) and the 55-CGE isodose line passing through the right optic nerve (right).

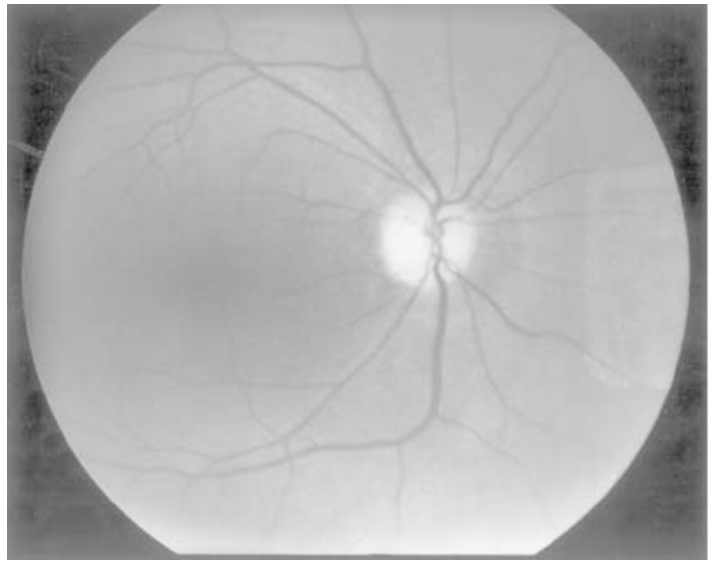

Figure 4 Fundal photographs showing bilateral optic atrophy.

rhinorrhoea. MRI revealed a tumour in the upper part of the clivus at the right petrous apex with invasion into the sphenoid sinus. Of particular note, 10 years previously she had developed a marked right sixth nerve palsy, undergoing a Jensen's transposition procedure. No imaging was performed at that time. Systemic lupus erythrematosus had been diagnosed 20 years previously.

At surgery, a clivus chordoma was found to extend to the petrous apex, forward to the cavernous sinus, and posteriorly to the cerebellopontine angle and pons. Though further tumour was removed from the right sphenoid sinus through a transnasal approach, only a subtotal resection was possible.

Postoperatively, she was found to have a right seventh nerve palsy requiring lateral tarsorrhaphy and right hearing loss.

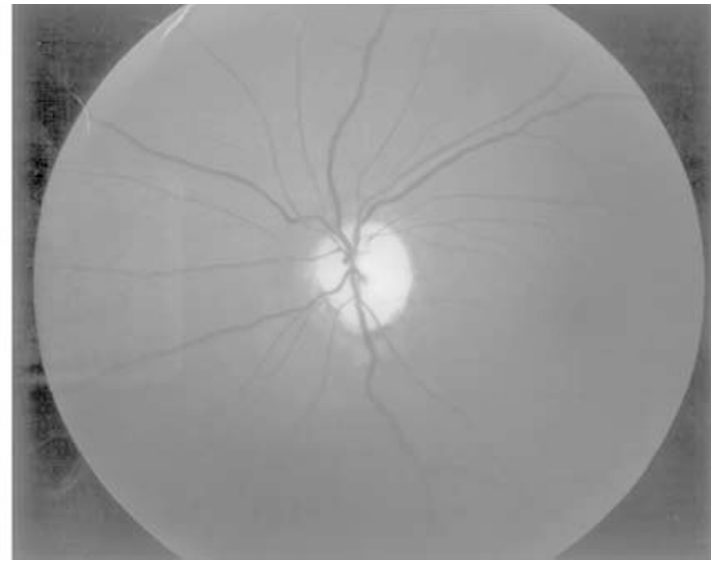

Residual tumour remained close to the brainstem. The patient was referred for proton/photon therapy to a dose of 75.8CGE (photon dose 14.8 Gy in eight fractions and proton dose of 61.4CGE in 32 fractions)

On ophthalmic review, now 3 years postproton beam therapy, best-corrected visual acuity remains right 6/6-3 and left 6/5-2 with normal colour vision. Optic discs were asymmetric, but normal colour with no relative afferent or field defect. No radiological recurrence of tumour or clinical progression has been noted.

\section{Case 4}

A 32-year-old man presented to the ophthalmologists with transient horizontal diplopia. He was found to have a microscopic, intermittant sixth nerve palsy. Computed 


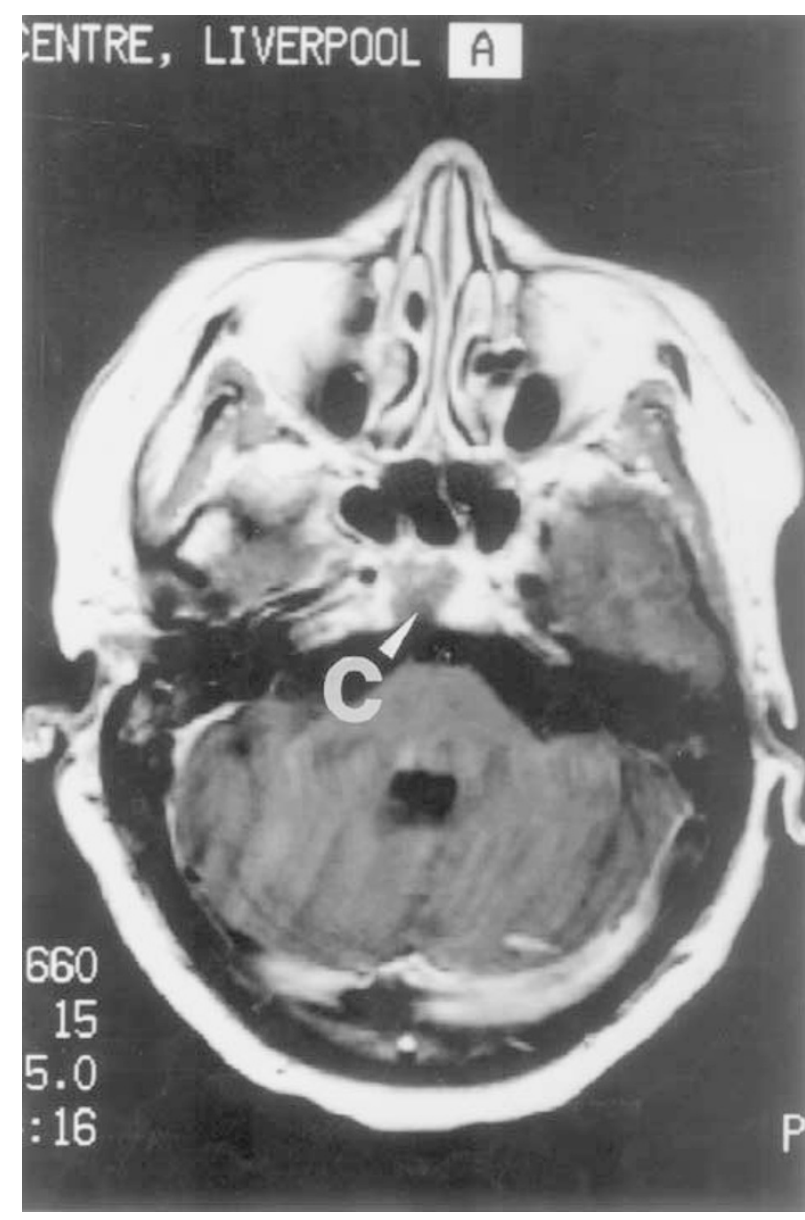

Figure 5 T1-weighted axial MRI scan with gadolinium enhancement showing postoperative residual tumour in the clivus (arrowed C).

tomography scan confirmed a prepontine tumour eroding the upper part of the clivus and posterior clinoids and extending to the suprasellar cistern. Angiography showed displacement of the basilar artery.

He subsequently underwent decompression of an extradural chordoma via a right temporal craniotomy and subtemporal exploration with removal of all macroscopic tumour. A mild right superior oblique weakness was noted postoperatively. Residual tumour was treated with proton/photon therapy to a dose of 72 CGE (photon dose 14.4 Gy in eight fractions and proton dose of 57.6CGE in 32 fractions). Two years later a significant radiation-induced hypopituitarism developed.

Three years later, a recurrence of the chordoma was diagnosed. Surgical decompression resulted in right sixth and seventh nerve palsies. Further proton beam therapy was not felt appropriate. It was recognised that a high

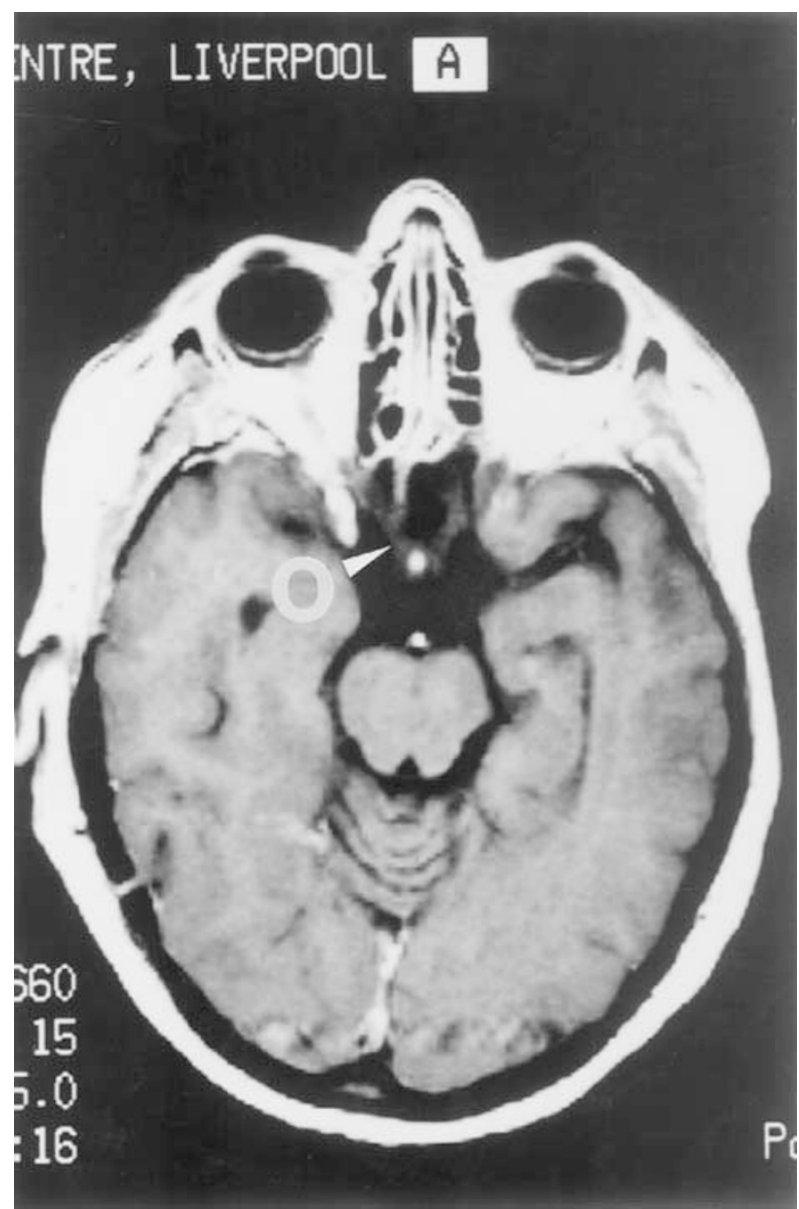

Figure 6 T1-weighted axial MRI scan with gadolinium enhancement showing the optic nerves and chiasm free of tumour (arrowed O) prior to proton beam radiotherapy.

radiation dose to the optic apparatus could risk subsequent blindness. Strabismus surgery was performed. Visual acuity was recorded most recently as right 6/9-3 and left 6/5, despite the presence of right inferior corneal scarring related to exposure.

\section{Discussion}

Symptoms and signs of clival chordoma reflect specific sites of tumour extension and may result in presentation to the ophthalmologist. In $60-90 \%$ of the cases, the initial complaint is of diplopia, usually from a sixth nerve palsy. A total of $25 \%$ will have a field defect or loss of acuity and $12 \%$ have optic disc swelling; $50 \%$ have symptoms or signs attributable to involvement of the 7-12th cranial nerves, cerebellum, or pons; $50 \%$ will complain of headache; $25 \%$ have symptoms of nasal involvement; and $12 \%$ have symptoms of pituitary involvement. ${ }^{2}$ 


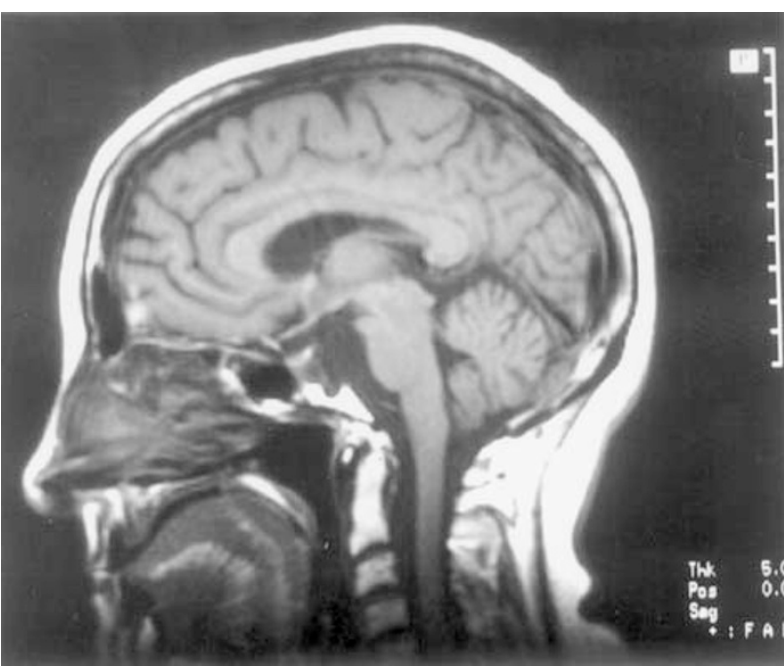

Figure 7 T1-weighted, unenhanced sagital MRI scan showing no recurrence of the tumour following development of optic neuropathy.

Since its first description in 1856 by Luschka, ${ }^{3}$ the management of chordomas has remained a particular challenge. If untreated, there is an average survival period between 0.6 and 2 years. ${ }^{2}$ Treatment options have included extensive surgical resection, external beam radiotherapy alone, or a combination of the two with average survival times of $1.5,4.8$, and 5.2 years, respectively. ${ }^{2}$

Complete resection, however, is nearly impossible and because of tumour insensitivity, high radiotherapy doses between 50 and 80 Gy are reported necessary to achieve palliation. ${ }^{4}$ Above $50 \mathrm{~Gy}$, postradiation injury to the optic nerves, chiasm, or tracts has been reported. In all, 52 clinically documented cases over 20 years following external fractionated radiotherapy were found in the literature by Habrand. ${ }^{5}$ The risk of developing radiation optic neuropathy was estimated at about 5-10\%, highest in the first 2 years but ranging from 2 months up to 8 years. ${ }^{5}$

Proton beam therapy gained interest in the 1980s because the proton 'Bragg peak' allowed a rapid reduction in dose distal to the target with a sharply defined lateral beam edge. Delivery of high doses of radiotherapy to residual tumour was reported possible with sparing of adjacent neurological structures. ${ }^{5-7}$

Proton energy deposition shows a shift towards higher lineal energy for proton beams relative to cobalt- 60 gamma rays, leading to a slightly elevated biological response. The relative biological effectiveness of protons to photons is $1.1 .^{8}$

Up to 76CGE was first reported delivered without significant morbidity in 1982 in two patients with clival chordoma. The technique used a combination of high- energy $10 \mathrm{MV}$ X-rays (photons) and $160 \mathrm{MeV}$ fixed horizontal proton beams. ${ }^{6}$

Neurovisual outcome was soon reported in 15 patients treated with a tumour of the upper clivus and followed up over 30-68 months. ${ }^{5}$ Doses of radiation received were estimated using a dose-volume histogram. When a fifth or more of the optic nerve was exposed to 60 CGE, a 22\% complication rate was estimated. At $55 \mathrm{CGE}$, the incidence was reduced to $12.5 \%$ for the optic nerve and $7.5 \%$ for the chiasm. Two patients who developed a progressive visual deterioration affecting both eyes were described. In the first, complete blindness in the right eye with a left temporal hemianopia developed 10 months following 68.9 CGE using exclusively protons. In the second, 3 years following a 66.58 CGE combination of protons / photons, visual acuities fell to NPL right and CF left. This patient had longstanding diabetes mellitus and had received a calculated dose to the chiasm of 60 CGE.

Pre-existing vascular disease, pituitary irradiation, concomitant chemotherapy, and a dose per session greater than $2.5 \mathrm{~Gy}$ were recognised as visual prognostic factors. ${ }^{5}$

Yet in all of our cases, either hypertension, postradiation hypopituitarism, or collagen vascular disease was present. None had chemotherapy. However, two went on to develop bilateral optic neuropathy and two did not.

In another report, a further patient developed unilateral blindness 9 months after receiving 70 CGE for a parasellar chondrosarcoma. ${ }^{1}$ Bilateral loss of vision developing concurrently with radiographically proven tumour progression in the upper clivus has been described..$^{9}$ There has been no evidence of this in our patients.

Temporal lobe damage, including seizures, are other complications of proton beam therapy that have been previously noted. The low incidence of treatment-related morbidity from proton beam therapy for clivus chordoma is felt to be within acceptable limits.

By 1999, optic neuropathy had developed in 12 of the 274 treated patients $(4.4 \%)$. Prescribed tumour doses ranged from 63.4 to 79.4CGE and the median dose to the optic structures in injured patients was 62.1CGE. ${ }^{10}$

Both of our patients with visual loss were female receiving tumour doses of 75 and 83.5CGE. These proton therapy doses are in accordance with the Proton Radiation Oncology Group Trial (PROG 85-26 Study). ${ }^{10}$ Preliminary findings had suggested a higher risk for female chordoma patients who were randomised to receive either 75 or 82.9 CGE. Notably at 82.9 CGE, normal tissue dose constraints were increased by $5 \%$. The resulting complications have been dramatic.

The proximity of the target field to the optic apparatus in our patients may have been a factor in the poor 
outcome. One patient had residual disease in the clivus and cavernous sinuses (Case 2) and the other in the upper clivus (Case 1), whereas the third and fourth cases had residual disease in the brainstem and anterior pons.

Although prescribed doses and fractionation schedules developed for conventional radiotherapy are similar for proton beam therapy, it is important to note that there are significant differences from photons in the physical interactions of protons with tissues, affecting the clinical use of the beam. Application of a different relative biological effectiveness for each different clinical tissue involved in the treatment area remains beyond the current state of the art in proton beam therapy. ${ }^{8}$ This will have important implications for a nonregenerative organ such as the optic pathway. A recent review of protocols for proton therapy in the USA observed major complications of unilateral or bilateral blindness, brain and brainstem necrosis in $8 \%$ of cases. ${ }^{11}$

Although the risk to visual pathways is reported to be low, ${ }^{10}$ we have experienced an unusually high rate of delayed, severe, and bilateral optic neuropathy. This may well represent an unfortunate coincidence. Our case series of four patients is too small to comment conclusively on factors influencing visual prognosis. However, we consider that currently, with the use of proton particle irradiation in such high doses, close to the optic chiasm and optic nerves there is a significant risk of developing the complications we have encountered. Proton beam therapy remains an expensive, timeconsuming, and demanding treatment option in the management of clival chordoma. We would like to highlight the potential hazard to the optic apparatus.

\section{References}

1 Austin-Seymour M, Munzenrider J, Goiten M, Varhey L, Urie M, Gentry $\mathrm{R}$ et al. Fractionated proton radiation therapy of chordoma and low-grade chondrosarcoma of the base of the skull. J Neurosurg 1989; 70: 13-17.

2 Heffelfinger J, Dahlin D, MacCarty C, Beabout J. Chordomas and cartilaginous tumours at the skull base. Cancer 1973; 32(2): 410-420.

3 Luschka H. Die Altersveranderungen der Zwischenwirbelknorpel. Virchows Arch Pathol Anat Physiol Klin Med 1864; 31: 396-399.

4 Gay E, Sekhar L, Wright D. Chordomas and chondrosarcomas of the cranial base. In: Kaye AH, Laws Jr ER (eds). Brain Tumors. Churchill Livingstone: New York, 1995, pp 777-794.

5 Habrand J, Austin Seymour M, Birnbaum S, Wray S, Carroll $\mathrm{R}$, Munzenrider J et al. Neurovisual outcome following proton radiation therapy. Int J Radiat Oncol Biol Phys 1989; 16(6): 1601-1606.

6 Suit HD, Goiten M, Munzenrider J et al. Definitive radiation therapy for chordoma and chondrosarcoma of base of skull and cervical spine. J Neurosurg 1982; 56: 377-385.

7 Austin JP, Urie MM, Cardenosa G, Munzenrider JE. Probable causes of recurrence in patients with chordoma and chondrosarcoma of the base of the skull and cervical spine. Int J Radiat Oncol Biol Phys 1993; 25(3): 439-444.

8 Miller DW. A review of proton beam radiation therapy. Med Phys 1995; 22(11): 1943-1954.

9 Hug EB, Loredo LN, Slater JD, DeVries A, Grove R, Schaefer $\mathrm{R}$ et al. Proton radiation therapy for chordomas and chondrosarcomas of the skull base. J Neurosurg 1999; 91: 432-439.

10 Munzenrider JE, Liebsch NJ. Proton therapy for tumours of the skull base. Strahlenther Onkol 1999; 175s: 57-63.

11 Krengli M, Liesbach NJ, Hug EB, Orecchia R. Review of current protocols for protontherapy in USA. Tumori 1998; 84: 209-216. 